\title{
Intelligent Decision Support for Improving Power Management
}

\author{
Yongqing Zheng, ${ }^{1,2}$ Han Yu, ${ }^{3,4}$ Kun Zhang, ${ }^{1,2,5}$ Yuliang Shi, ${ }^{1,2}$ Cyril Leung, ${ }^{4,6}$ and Chunyan Miao ${ }^{3,4}$ \\ ${ }^{1}$ School of Software, Shandong University \\ ${ }^{2}$ Dareway Software Co. Ltd \\ ${ }^{3}$ School of Computer Science and Engineering, Nanyang Technological University \\ ${ }^{4}$ Joint NTU-UBC Research Centre of Excellence in Active Living for the Elderly \\ ${ }^{5}$ School of Information Science and Engineering, University of Jinan \\ ${ }^{6}$ Department of Electrical and Computer Engineering, The University of British Columbia \\ zhengyongqing@dareway.com.cn, han.yu@ntu.edu.sg, shiyuliang@sdu.edu.cn
}

\begin{abstract}
With the development and adoption of the electricity information tracking system in China, realtime electricity consumption big data have become available to enable artificial intelligence (AI) to help power companies and the urban managemen$\mathrm{t}$ departments to make demand side managemen$\mathrm{t}$ decisions. We demonstrate the Power Intelligent Decision Support (PIDS) platform, which can generate Orderly Power Utilization (OPU) decision recommendations and perform Demand Response (DR) implementation management based on a short-term load forecasting model. It can also provide different users with query and application functions to facilitate explainable decision support.
\end{abstract}

\section{Introduction}

Artificial intelligence (AI) has become pervasive in today's world. It is changing many aspects of our life, including the e-government [Zheng et al., 2018], healthcare [Sun et al., 2019], environment protection [Pan et al., 2017], social networking [Shen et al., 2011], and finance [Ren et al., 2019]. Efficient management of electric power on a societal level plays a vital role in economic development, national security, and social stability. In recent years, with in-depth power market reform and the deployment of smart grids in China, users are enjoying increasing flexibility in electricity consumption. Efficient power demand side management and effective power dispatch will significantly improve the wellbeing of a city. Accurate, short-term power load forecasting is the basis for demand side power management, and affects the optimization of Orderly Power Utilization (OPU) [Srivastava et al., 2016] and Demand Response (DR) [Strasser et al., 2015].

Short-term load forecasting has been an important topic of research in smart grid. Popular existing load forecasting methods include [Hippert et al., 2001], regression analysis [Stulp and Sigaud, 2015], support vector machine and artificial neural networks [Kong et al., 2019]. More recent works are increasingly focused on time series and combined forecasting models to perform forecasting. These methods have proven to be more effective as they can better handle the temporal characteristics of the load data.

In this paper, we demonstrate the Power Intelligent Decision Support (PIDS) platform. It adopts a novel short-term load forecasting model (Wavelet Decomposition and Long Short-Term Memory model, WD-LSTM for short), which combines influencing factor analysis, wavelet decomposition feature extraction, triple order exponential smoothing (HoltWinters) time series analysis, and Long Short-Term Memory (LSTM) networks. The model has been shown to significantly improve prediction accuracy.

PIDS provides users with the function of querying historical power consumption and electricity usage analysis results. Users can participate in Orderly Power Utilization (OP$\mathrm{U})$ and Demand Response (DR) processes through this system. The urban management authority can also use this system to make optimal control decisions [Pan et al., 2016], and manage the OPU and DR processes with explainable decision support based on the load forecasting results produced by WD-LSTM. It can be implemented as an interactive decision support agent [Yu et al., 2007; 2010; 2011; Lin et al., 2015].

\section{Platform Overview}

The overall architecture of the platform is shown in Figure 1. It comprises of four tiers, including data collection tier, modeling tier, utility tier and Interaction tier.

The data collection tier aggregates related data from multiple sources. These include load data and user profile information from the Electricity Information Collection System, Meteorological data from the China Meteorological Data Service Center, and holiday data from public holiday calenders. The Modeling tier that performs short-term load forecasting. Load forecasting is the basis for subsequent power demand side management. Its accuracy will affect the effectiveness of subsequent steps. The proposed WD-LSTM model can meet the accuracy need for our purpose. The Utility tier includes DR and OPU. Based on the results of short-term load forecasting, the relevant authority determines the time for DR invitations and the optimal power usage decisions for OPU.

In Interaction tier, users can query their historical power consumption and electricity usage behavior analysis reports 


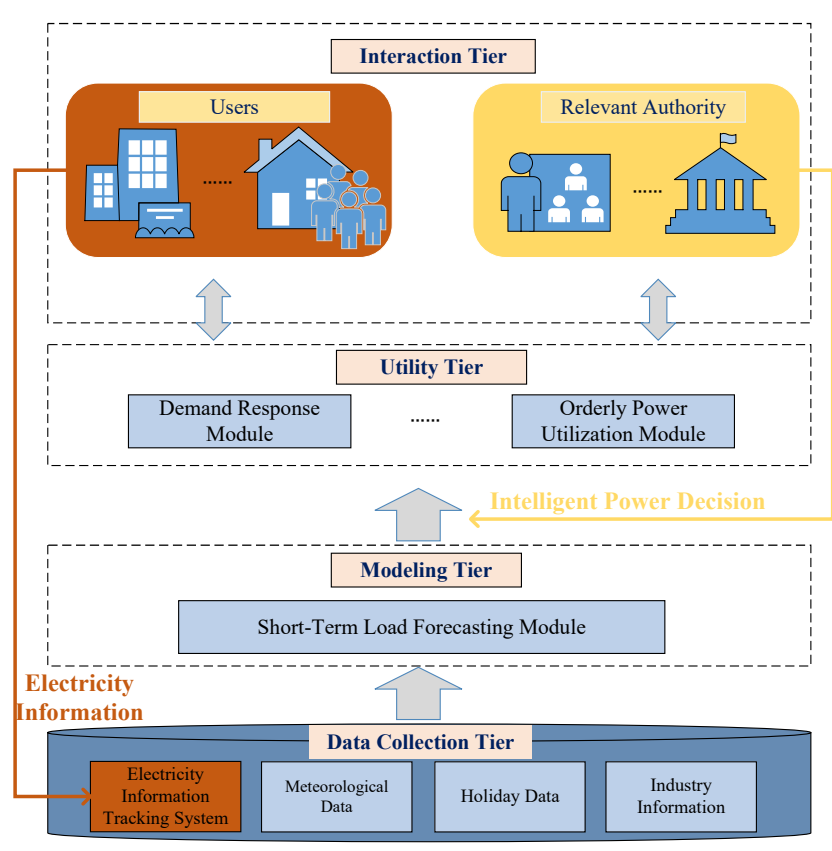

Figure 1: The architecture of the PIDS platform.

through interactions with the system. They can also participate in the DR invitation and OPU through the system. The power dispatch results produced by the demand side management are delivered to the Electricity Information Collection System as feedbacks. The relevant authority can audit the processes of OPU and DR through the system based on human interpretable explanations generated by the AI engine, and adjust the next round of work plan based on these results.

\section{The AI Engine}

\subsection{Short-Term Load Forecasting}

A novel short-term load forecasting model - WD-LSTM - is adopted by the PIDS platform. It combines influencing factor analysis, wavelet decomposition feature extraction, triple order exponential smoothing (Holt-Winters) time series analysis and Long Short-Term Memory (LSTM) networks. The model uses wavelet decomposition to extract the main features of load data, analyzes its correlation with temperature, holidays and industry influencing factors, and then construct$\mathrm{s}$ corresponding adjustment factors. The Holt-Winters algorithm is used to forecast each feature in subsequent time steps. The preliminary forecasting results and the adjustment factors are passed on to the LSTM networks to perform regression fitting. Finally, the forecasting results are generated through wavelet inverse transformation.

\subsection{Intelligent Demand Response (DR)}

When the platform forecasts that there will be a peak load in the immediate future based on the short-term forecast results, the DR invitation operation is executed. The users query the historical baseline and electricity usage amount, and then decide whether to participate in response to the actual situation.
For participating users, the platform analyzes their responsiveness according to their historical power consumption and response data. Then, the platform selects users according to preset requirements. DR is executed during specified time periods, and the platform monitors the users' response status in real time.

\subsection{Intelligent Orderly Power Utilization (OPU)}

In the preparation stage of OPU, according to the prediction of possible overall gaps, the platform develops diverse load control schemes for non-residential households, speciallimited households, large-scale users, etc. Evaluating the peak period according to short-term load forecasting results. Then, the corresponding optimal control policy is selected by the platform to be execution, with detailed monitoring of the execution effect.

\subsection{Platform Implementation}

The power decision intelligent support (PIDS) platform is built on Hadoop for storing the big data in power consumption. The data visualization interface for the power management authority to use is shown in Figure 2. Privacypreserving decision support capabilities based on federated machine learning [Yang et al., 2019] will be incorporated in future to enable collaboration across power companies in compliance with privacy laws.

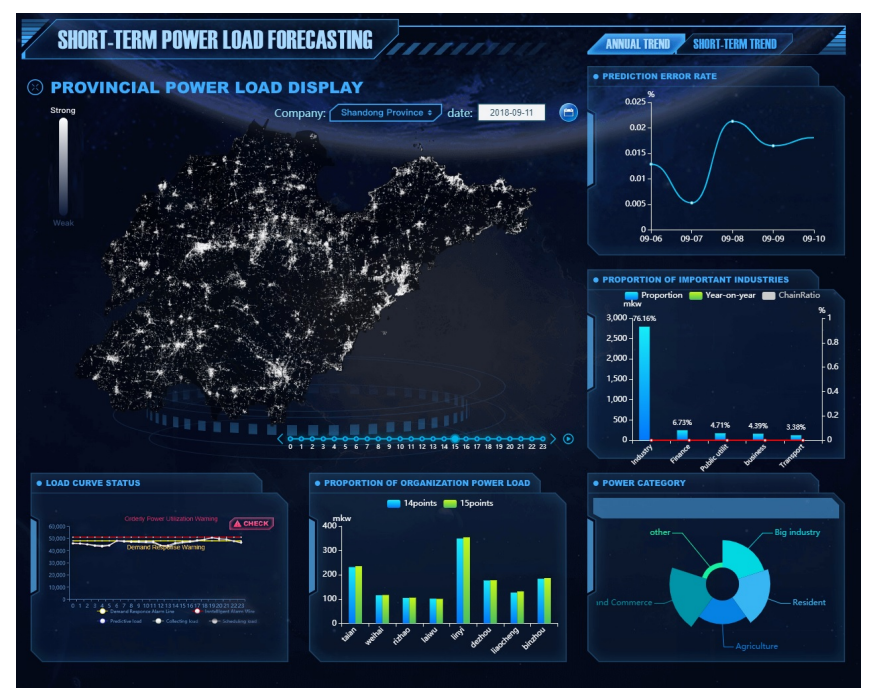

Figure 2: An example PIDS user interface.

\section{Acknowledgements}

This research is supported, in part, by the National Key Research and Development Plan of China (No.2018YFB1003804), the Taishan Industrial Experts Program of Shandong Province of China (No.tscy20150305), the Key Research \& Development Program of Shandong Province of China (No.2016ZDJS01A09), and Nanyang Technological University, Nanyang Assistant Professorship (NAP). 


\section{References}

[Hippert et al., 2001] Henrique S. Hippert, Carlos E. Pedreira, and Reinaldo C. Souza. Neural networks for shortterm load forecasting: a review and evaluation. IEEE Transactions on Power Systems, 16(1):44-55, 2001.

[Kong et al., 2019] Weicong Kong, Zhao Yang Dong, Youwei Jia, David J. Hill, Yan Xu, and Yuan Zhang. Shortterm residential load forecasting based on LSTM recurrent neural network. IEEE Transactions on Smart Grid, 10(1):841-851, 2019.

[Lin et al., 2015] Han Lin, Jinghua Hou, Han Yu, Zhiqi Shen, and Chunyan Miao. An agent-based game platform for exercising people's prospective memory. In WI-IAT, pages 235-236, 2015.

[Pan et al., 2016] Zhengxiang Pan, Han Yu, Chunyan Miao, and Cyril Leung. Efficient collaborative crowdsourcing. In $A A A I$, pages 4248-4249, 2016.

[Pan et al., 2017] Zhengxiang Pan, Han Yu, Chunyan Miao, and Cyril Leung. Crowdsensing air quality with cameraenabled mobile devices. In IAAI, pages 4728-4733, 2017.

[Ren et al., 2019] Rui Ren, Desheng Dash Wu, and Tianxiang Liu. Forecasting stock market movement direction using sentiment analysis and support vector machine. IEEE Systems Journal, 13(1):760-770, 2019.

[Shen et al., 2011] Zhiqi Shen, Han Yu, Chunyan Miao, and Jianshu Weng. Trust-based web service selection in virtual communities. Web Intelligence and Agent Systems, 9(3):227-238, 2011.

[Srivastava et al., 2016] A. K. Srivastava, Ajay Shekhar Pandey, and Devender Singh. Short-term load forecasting methods: A review. In International Conference on Emerging Trends in Electrical Electronics and Sustainable Energy Systems, 2016.

[Strasser et al., 2015] Thomas Strasser, Filip Andrn, Johannes Kathan, Carlo Cecati, Concettina Buccella, Pierluigi Siano, Paulo Leito, Gulnara Zhabelova, Valeriy Vyatkin, and Pavel Vrba. A review of architectures and concepts for intelligence in future electric energy systems. IEEE Transactions on Industrial Electronics, 62(4):24242438, 2015.

[Stulp and Sigaud, 2015] Freek Stulp and Olivier Sigaud. Many regression algorithms, one unified model: A review. Neural Networks, 69:60-79, 2015.

[Sun et al., 2019] Chenfei Sun, Qingzhong Li, Hui Li, Yuliang Shi, Shidong Zhang, and Wei Guo. Patient cluster divergence based healthcare insurance fraudster detection. IEEE Access, 7:14162-14170, 2019.

[Yang et al., 2019] Qiang Yang, Yang Liu, Tianjian Chen, and Yongxin Tong. Federated machine learning: Concept and applications. ACM Transactions on Intelligent Systems and Technology, 10(2):12:1-12:19, 2019.

[Yu et al., 2007] Han Yu, Zhiqi Shen, and Chunyan Miao. Intelligent software agent design tool using goal net methodology. In IAT, pages 43-46, 2007.
[Yu et al., 2010] Han Yu, Yundong Cai, Zhiqi Shen, Xuehong Tao, and Chunyan Miao. Agents as intelligent user interfaces for the net generation. In IUI, pages 429-430, 2010.

[Yu et al., 2011] Han Yu, Zhiqi Shen, Chunyan Miao, and Ah-Hwee Tan. A simple curious agent to help people be curious. In $A A M A S$, pages 1159-1160, 2011.

[Zheng et al., 2018] Yongqing Zheng, Han Yu, Lizhen Cui, Chunyan Miao, Cyril Leung, and Qiang Yang. SmartHS: An AI platform for improving government service provision. In IAAI, pages 7704-7712, 2018. 